\title{
Influence of pectinase-assisted extraction time on the antioxidant capacity of Spent Coffee Ground (SCG)
}

\begin{abstract}
Spent Coffee Ground (SCG) comprises a range of functional components with high antioxidant potential and health benefits. Although SCG has many advantages, antioxidant values obtained using enzymes is still scarce. Several studies reported that the use of enzyme-assisted extraction techniques could improve the detection of antioxidant compounds in samples of by-products. In this study, SCG was extracted using enzymeassisted techniques for different periods $(0,20$, 40, 60, 80 and 100 mins). The total antioxidant capacity was measured using Peroxidase, Catalase, Ferric reducing antioxidant power (FRAP), Superoxide radical scavenging assay (SRSA) and 2,2-diphenyl-2- picrylhydrazyl (DPPH) analysis. 60 mins extraction was found to exhibit antioxidant activity in DPPH, SRSA, Catalase and Peroxidase of $84.18 \pm 7.01 \%$, $32.83 \pm 6.75 \%, 76.65 \pm 5.52 \%$ and $75.01 \pm 7.67 \%$, respectively. Besides that, the extraction also gave a high amount of content with $267.17 \pm 26.69 \mathrm{mg} \mathrm{TAE} / \mathrm{mL}$ and $58.30 \pm 2.36 \mathrm{mg} \mathrm{QE} / \mathrm{g}$ in total phenolic content and total flavonoid content respectively. Flavonoids such as quercetin, kaempferol, rutin, gallic acid, catechin, epigallocatechin, p-Coumaric acid and myricetin were found to be present in SCG extract. A high amount of catechin was found in all periods of extraction, whereby $60 \mathrm{mins}$ had the highest concentration of $1741.65 \mathrm{mg} / \mathrm{L}$, while $40 \mathrm{mins}$ had the lowest concentration which was $389.85 \mathrm{mg} / \mathrm{L}$. In conclusion, $60 \mathrm{mins}$ extraction using a pectinase-assisted method was the best extraction period. Additionally, the individual flavonoid catechin in SCG showed that it has potential as an antioxidant. Finally, there is a strong correlation between antioxidative activity and both phenolic and flavonoid content.
\end{abstract}

Keyword: Spent coffee ground (SCG); Pectinase-assisted extraction; Antioxidative activities; Antimicrobial activities 